\title{
Stability analysis of milling with irregular pitch tools by the implicit subspace iteration method
}

\author{
Mikel Zatarain • Zoltan Dombovari
}

Received: 24 September 2013 / Revised: 24 November 2013 / Accepted: 11 December 2013 / Published online: 15 January 2014 (C) Springer-Verlag Berlin Heidelberg 2014

\begin{abstract}
Irregular pitch tools have been used since a long time ago to avoid chatter in milling processes. Optimal design of these mills was studied by some researchers. The method used is based in the frequency domain, and there were some practical examples showing that the process gives good results. Nevertheless, for high order lobes the method was not verified analytically, one of the difficulties being the large size of the matrices involved in the analysis. A recent method for obtaining stability diagrams is based in the use of implicit subspace iteration method, giving rise to much shorter calculation times than the already known discretized time domain methods. Use of this method allowed assessing the stability of processes with irregular pitch mills in both high and medium order lobes regions. The method of subspace iteration was compared with the more conventional semi-discretization method in low order lobes region, with good agreement. Afterwards, the stability of milling processes with irregular pitch tools designed after previous proposals was assessed in both medium and high order lobes regions. As a conclusion, in the examples analyzed the angles selection shows to be an optimal solution, although for high order lobes the regular pitch tools provides better stability. As a further research topic, other possible angle distributions to improve the behavior at low velocities should be analyzed.
\end{abstract}

Keywords Chatter - Irregular pitch tools · Milling ·

Tool design

\section{Zatarain}

Dynamics and Control, Ideko-IK4, 20870 Elgoibar,

Gipuzkoa, Spain

e-mail: mzatarain@ideko.es

\section{Z. Dombovari ( $\varangle)$}

Department of Applied Mechanics, Budapest University of Technology and Economics, Budapest 1521, Hungary

e-mail:dombo@mm.bme.hu

\section{Introduction}

Chatter vibration is a main limitation for the productivity of machining processes. Chatter gives rise to high vibration level, bad part surface, and reduced life of tools and machine components. There are many strategies to avoid chatter in milling processes, which are more or less suited depending on the particular application. One of the solutions proposed is the use of uneven pitch tools, which disrupt the regeneration of chatter vibration giving rise to extended cutting capability.

Selection of the best possible angle between cutting teeth is an issue which has been analyzed in some previous papers. Budak [1,2] proposed a method to optimize stability at a given rotation speed for a single mode system, while Suzuki et al. [3] extended the solution for two mode systems. The optimization was based on a simplified analysis in the frequency domain, by considering the zeroth order approach, that is, disregarding the effect of the harmonics of the main vibration frequency. Several applications were shown with good results for the optimized variation of pitch, but the stability diagrams were not calculated by the more accurate multi-frequency solution.

Time domain methods have also been applied to verify the stability of milling processes with uneven pitch mills. The stability analysis is done by calculating the well-known lobe diagram. There are several methods of this kind, which have the advantage over frequency domain to be very robust and to consider all the harmonics of the main frequency, but at the same time they do not provide the capacity of analyzing the phenomenon from an engineering point of view. One drawback of these time domain based methods is the limitations when calculating the stability at low cutting frequencies (high order lobes of the stability diagram), due to the large size of the matrices involved. 
The goal of the work being presented here is to obtain such verification for some examples which were proposed in the bibliography by a time domain method that is much faster computationally than the already known methods.

The paper will start by a revision of the state of the art. Then, the way of applying the implicit subspace iteration method (ISIM) to obtain stability prediction in milling processes will be shown. Finally, the method will be validated by resolution of already published cases, and stability diagrams with the tool pitch variations proposed by the design method by $[1,2]$ will be obtained.

\section{State of the art}

The first theories explaining the uprising of chatter as a regenerative phenomenon were developed by Tobias and Fishwick [4] and by Tlusty and Polacek [5]. Later, Merritt [6] interpreted the system as a feedback process. As a geometric approach of the system, the directional factor describes the relationship between the cutting force and the modal displacement, and between the modal displacement and the chip thickness. For milling processes, where the geometry of the system varies, the system is much more complicated to be solved than in the case of simple cutting geometry, as in the case of turning. Opitz and Bernardi [7] proposed a way of calculating an equivalent directional factor. By means of it, limit depth of cut diagrams for milling, for one degree of freedom systems, could be obtained.

A more precise solution for the stability assessment of the milling process was formulated by Minis et al. [8], by using Floquets theorem [9] and Fourier series, and determining the stability limits numerically using the Nyquist stability criterion. Budak and Altintas [10] extended this approach by proposing an analytical method for the stability prediction of milling, giving rise to a multi-frequency analysis, in which chatter vibration has several frequency components separated by the cutting frequency. When only the first term of the frequency content of the vibration is considered the system can be solved in an extremely fast way [11]. For a system with a single degree of freedom, the result coincides with the solution proposed by Opitz and Bernardi [7].

Other developments by Davies et al. [12], Insperger et al. [13,14] and Bayly et al. [15] obtained the stability limits using different methods in the time domain, by separating the solution between the forced part and the stability problem, giving rise to an eigenvalue system. All these methods mentioned above performed the analysis in the Cartesian coordinates system, with the limitation of having modal displacements in the Cartesian directions. While this approach might be acceptable for high frequency chatter in milling processes, which usually is due to the flexibility of the spindle and of the tool, when instability is due to structural vibration this simplification cannot be accepted. Zatarain et al. [16] analyzed the effect of the feed direction with respect to the modal displacement direction by working in modal coordinates, formulation that is valid for structural instability also.

Fitting chatter was done by many different approaches. It is possible to mention the use of high damping structural materials like polymer concrete [17], dynamic vibration absorbers [18], impact absorbers [19], continuous variation of spindle speed [20], and so on.

One of the ways to get rid of chatter in milling is the use of uneven pitch tools. Right selection of the pitch angles requires the use of models of the process with uneven pitch mills. It is worth to mention the papers by Slavicek [21], Opitz [22], Vanherck [23], and Tlusty et al. [24], who analyzed the stability of milling processes with irregular pitch tools by simplified approaches. Budak [1,2] proposed an approach for the selection of the pitch variation. The analysis was done in the frequency domain by the zero order approach (single frequency). Working with methods in the time domain stability diagrams were shown in several papers $[25,26]$. The limitation of these methods is the size of the transition matrix when high order lobes region has to be analyzed, as the complete tool rotation has to be discretised instead of the tooth pass angle. The subspace iteration method was proposed as a way to speed up the solution of time domain methods, and at the same time to allow working with much higher size matrices. This method seems very suitable to cope with the problem of high order lobe stability with uneven pitch mills.

It is also possible one performs time-domain simulations of complex milling processes based on standard solvers like dde23 in Matlab [27]. However, one can realize the presented method can also cope with these standard solvers only having one period simulations rather than long transient simulations to decide the stability of stationary cutting.

Therefore, the goal of the research is the use of the subspace iteration method to assess the stability of processes with irregular pitch tools, and to evaluate the analytical results when the pitch angles are selected following the simplified analysis $[1,2]$.

\section{Method of subspace iteration applied to analyze milling stability}

Time domain based methods to predict the stability of a milling process need the calculation of the transition matrix, relating the state at a particular moment with the state one period later, and then the calculation of the dominant eigenvector/eigenvalue pair of that matrix. The magnitude of the eigenvalue being higher or lower than 1 defines if the system is respectively unstable or stable.

The subspace iteration method for assessing stability of milling can be explained in two steps: solving the eigenvalue 
problem by the subspace iteration method, which requires calculation of the product of the transition matrix by guess vectors a number of times, and then the way to obtain this product without the need to explicitly obtain the transition matrix.

\subsection{General method for reduction of eigenproblem size}

The different methods for computing eigenvalues $\boldsymbol{\mu}=$ $\operatorname{diag}_{k=1}^{n} \mu_{k}$ and eigenvectors $\mathbf{S}=\operatorname{row}_{k=1}^{n} \mathbf{s}_{k}$ can be considered as variants of power iteration for the same eigenvalue problem described as

$\mathbf{Z S}=\mathbf{S} \boldsymbol{\mu}$,

where $n$ denotes the size of the square transition matrix $\mathbf{Z}$. If $\mathbf{S}_{j}=\operatorname{row}_{k=1}^{N_{s}} \mathbf{S}_{j, k}$ is the $j$ th guess for the set of $N_{s} \leq n$ dominant eigenvectors, and a power iteration step is applied to this vector set, a new set $\mathbf{V}_{j}$ will be obtained

$\mathbf{V}_{j}=\mathbf{Z} \mathbf{S}_{j}$.

If $\mathbf{S}_{j}$ is a set obtained after several steps of power iteration it can be considered that the vectors in this set will have strong content of the dominant eigenvectors, and a low content of the less dominant ones. Therefore, if the basis formed by the vectors in $\mathbf{S}_{j}$ and the vectors in $\mathbf{V}_{j}$ expand approximately the same space, we can obtain a matrix $\mathbf{H}_{j}$ which approximately relates both vector sets in the minimum square error sense, that is

$\mathbf{V}_{j} \approx \mathbf{S}_{j} \mathbf{H}_{j}, \quad$ and $\mathbf{H}_{j}=\left(\mathbf{S}_{j}^{\top} \mathbf{S}_{j}\right)^{-1} \mathbf{S}_{j}^{\top} \mathbf{V}_{j}$.

If we solve the eigenvalue and eigenvector problem on matrix $\mathbf{H}_{j}$ we will have

$\mathbf{H}_{j}=\mathbf{G}_{j} \lambda_{j} \mathbf{G}_{j}^{-1}$.

For the sake of the next iteration step we can state

$\mathbf{S}_{j} \approx \mathbf{S} \mathbf{Q}_{j}$,

since $\mathbf{S}_{j}$ contains guess of the eigenvectors related to the largest eigenvalues of the matrix $\mathbf{Z}$. In (5) $\mathbf{S}$ are the accurate corresponding eigenvectors. Then, the vectors $\mathbf{V}_{j}$ will be after (2) and (3)

$\mathbf{V}_{j} \approx \mathbf{S} \mathbf{Q}_{j} \mathbf{H}_{j}=\mathbf{S} \mathbf{Q}_{j} \mathbf{G}_{j} \lambda_{j} \mathbf{G}_{j}^{-1}=\mathbf{S} \boldsymbol{\mu} \mathbf{Q}_{j}$.

Then one can derive

$\mathbf{G}_{j} \lambda_{j} \mathbf{G}_{j}^{-1}=\mathbf{Q}_{j}^{-1} \boldsymbol{\mu} \mathbf{Q}_{j}$.

Then it is possible to deduct that the eigenvectors $\mathbf{G}_{j}$ of the matrix $\mathbf{H}_{j}$ represent the inverse of the content $\mathbf{Q}_{j}$ of the $j$ th guess of eigenvectors $\mathbf{S}_{j}$. Therefore, the exact eigenvector is theoretically

$\mathbf{S} \approx \mathbf{V}_{j} \mathbf{G}_{j} \lambda_{j}^{-1}$, after equation (6). As the matrix $\lambda_{j}$ is diagonal it only influences on the size of the columns of $\mathbf{S}$. Therefore, it is possible to disregard that value. The new iteration vector (guess of the eigenvectors) will be

$\mathbf{S}_{j+1}=\mathbf{V}_{j} \mathbf{G}_{j}$.

\subsection{Avoiding calculation of the transition matrix}

The next problem to deal with is that of the long calculation time required to form the transition matrix $\mathbf{Z}$ by any of the existing methods (semi-discretization, time finite elements, etc.). Therefore, an alternative possibility will be proposed here. The methods shown in the previous section for calculating the eigenvalues require processing multiplications of the matrix times a number of approximated state vectors in $\mathbf{S}_{j}$. If a method to calculate those products without the explicit use of the transition matrix is developed, it will not be necessary to form it, and therefore the time required to compute the stability diagrams might reduce significantly.

If we start with any initial solution for the state over a period length of time, we could calculate the situation one period $T$ later by multiplying the initial solution by the transition matrix; but another possibility is to perform the time integration over that period. The result of both methods must be the same, except for differences given by numerical errors, and if an efficient algorithm is used to perform the time integration, the result can be obtained much faster than the time required to form the transition matrix.

For example, if the Krylov subspace has to be obtained, starting from the same vector $\mathbf{s}_{0,1}$ in e.g. $\mathbf{S}_{0}$ at (2) instead of obtaining that subspace by forming the complete transition matrix $\mathbf{Z}$ and performing the matrix vector multiplications indicated in the previous section. It is possible to directly compute the time integration of the system response for the required number of periods. The vectors obtained after each of the periods are the vectors that have to be used to form the Krylov subspace, like $\mathbf{S}_{0}:=$ $\left[\begin{array}{lll}\mathbf{s}_{0,1} & \mathbf{Z} \mathbf{s}_{0,1} & \mathbf{Z}^{2} \mathbf{s}_{0,1} \ldots \mathbf{Z}^{N_{s}-1} \mathbf{s}_{0,1}\end{array}\right]$.

An efficient way to apply this alternative consists in calculating the step matrices, which relate the dynamic state at each of the discretized positions with the state at the previous position and with the mark left by the previous pass. As the state is a vector of very small size, these step matrices are very small.

\subsection{Calculation of step matrices in a milling process}

The milling process is described in the modal space with $m$ modal coordinates $\mathbf{q}(t):=\operatorname{col}_{l=1}^{m} q_{l}(t)$ which can be transformed back to spatial Cartesian space by using the mass normalized modal transformation matrix $\mathbf{U}$. Then

$\mathbf{r}(t)=\mathbf{U} \mathbf{q}(t)$, 
where $\mathbf{r}(t):=\operatorname{col}(x(t), y(t), z(t))$. The matrix $\mathbf{U}=$ $\left[\begin{array}{llll}\mathbf{u}_{1} & \mathbf{u}_{2} & \ldots & \mathbf{u}_{m}\end{array}\right]$ contains $m$ modeshapes, in which sense this matrix is a truncated modal matrix. In order to have a reasonable model of milling processes such mathematical model needs to developed that can handle multiple modes occasionally in the same direction and also keep the size of the problem as low as it is possible. Considering the regenerative effect between subsequent edges induces the past states to be activated the mathematical model is going to have delay differential equation (DDE) form. This means the system is infinite dimensional and from dynamic point of view it can be described in the following state space

$$
\left[\mathbf{q}_{t}(\theta) \dot{\mathbf{q}}_{t}(\theta)\right]^{\top},
$$

where the notation $\mathbf{q}_{t}(\theta)=\mathbf{q}(t+\theta)(\theta \in[-\sigma, 0])$ describes the continuum amount of involved past states up to $\theta=$ $-\sigma$. As later one can realize, past states only act through the resultant cutting force, which is compiled using the Cartesian coordinates $\mathbf{r}$ through the local chip thickness. This means in fact it is enough to use the following abstract mixed state

$\mathbf{z}_{t}(\theta):= \begin{cases}{[\mathbf{q}(t) \dot{\mathbf{q}}(t)]^{\top},} & \theta=0, \\ \mathbf{r}_{t}(\theta), & -\sigma \leq \theta<0,\end{cases}$

which is still infinite dimensional, but tracks only maximum three dimensional Cartesian delayed states rather than $2 m$ dimensional modal states considering modal velocities, too. Any discretization of (12) results the most efficient discrete state for any kind of calculation related to milling processes.

The chip thickness cut by the $i$ th edge segment at the axial level of $z$ can be expressed in the following form

$$
\begin{aligned}
h_{i}\left(z, t, \mathbf{r}_{t}(\theta)\right) \approx & \left(f_{i}+x(t)-x\left(t-\tau_{i}\right)\right) \sin \varphi_{i}(z, t) \\
& +\left(y(t)-y\left(t-\tau_{i}\right)\right) \cos \varphi_{i}(z, t),
\end{aligned}
$$

where the lead angle is considered as $\kappa=90^{\circ}$ and the angular displacement of the $i$ th flute at level $z$ can be expressed with

$\varphi_{i}(z, t)=\Omega t+\sum_{k=1}^{i-1} \varphi_{\mathrm{p}, k}-\varphi_{\eta}(z)$

Here $\eta$ and $\varphi_{\eta}(z)=\frac{2}{D} z \tan \eta$ are the helix and the lag angle, $D$ is the diameter of the tool and $\varphi_{\mathrm{p}, i}$ is the pitch angles between subsequent edges, which in fact determine the regeneration as (see Fig. 1)

$\tau_{i}=\frac{1}{\Omega} \varphi_{\mathrm{p}, i}$.

Having the regeneration time between subsequent edges the feed motion in (13) can be expressed as

$f_{i}=f \frac{\Omega}{2 \pi} \tau_{i}$,

where $f$ is the feed per revolution and $\Omega$ is the angular velocity of the tool. The theoretical feed per tooth can be defined as $f_{Z}=f / Z$, where $Z$ is the number of teeth. The calculated

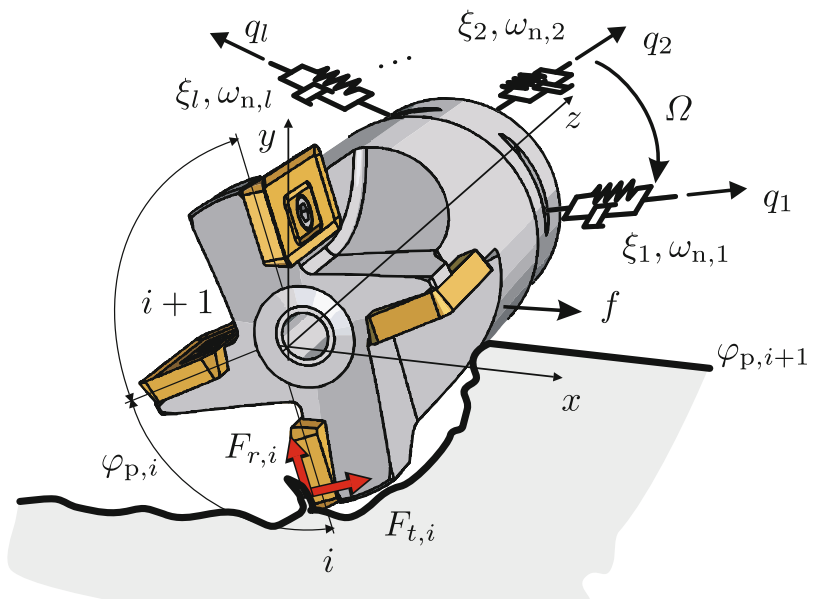

Fig. 1 Model of milling process

chip depth produces distributed cutting force on the $i$ th edge segment that can be calculated as:

$\mathbf{f}_{t r a, i}\left(z, t, \mathbf{r}_{t}(\theta)\right)=-\mathbf{K}_{\mathrm{e}}-\mathbf{K}_{\mathrm{c}} h_{i}\left(z, t, \mathbf{r}_{t}(\theta)\right)$,

where the edge coefficients and cutting coefficients are $\mathbf{K}_{\mathrm{e}}=$ $\left[\begin{array}{lll}K_{\mathrm{e}, t} & K_{\mathrm{e}, r} & K_{\mathrm{e}, a}\end{array}\right]^{\top}$ and $\mathbf{K}_{\mathrm{c}}=\left[\begin{array}{lll}K_{\mathrm{c}, t} & K_{\mathrm{c}, r} & K_{\mathrm{c}, a}\end{array}\right]^{\top}$. After integrating along the edges and summing all "edge" forces the resultant force can be expressed in Cartesian coordinate system

$$
\begin{aligned}
\mathbf{F}\left(t, \mathbf{r}_{t}(\theta)\right)= & -\frac{1}{\cos \eta} \sum_{i=1}^{Z} \int_{0}^{a_{\mathrm{p}}} g_{i}\left[\begin{array}{ccc}
\cos \varphi_{i} & \sin \varphi_{i} & 0 \\
-\sin \varphi_{i} & \cos \varphi_{i} & 0 \\
0 & 0 & 1
\end{array}\right] \\
& \times\left(\mathbf{K}_{\mathrm{e}}+\mathbf{K}_{\mathrm{c}} h_{i}\left(z, t, \mathbf{r}_{t}(\theta)\right)\right) \mathrm{d} z,
\end{aligned}
$$

where the screen function considers the radial immersion as

$g_{i}:=g_{i}(z, t)= \begin{cases}1, & \varphi_{\mathrm{en}} \leq \varphi_{i}(z, t) \bmod 2 \pi \leq \varphi_{\mathrm{ex}}, \\ 0, & \text { otherwise }\end{cases}$

and $\varphi_{i}:=\varphi_{i}(z, t)$ defined at (14). In (18) the $a_{\mathrm{p}}$ is the axial immersion of the cylindrical milling tool. In (19) $\varphi_{\mathrm{en}}$ and $\varphi_{\text {ex }}$ denote the enter and exit angles of the radial immersion. The resultant force excites the dynamics described in modal space in the following way

$\ddot{\mathbf{q}}(t)+\left[2 \xi_{l} \omega_{\mathrm{n}, l}\right] \dot{\mathbf{q}}(t)+\left[\omega_{\mathrm{n}, l}^{2}\right] \mathbf{q}(t)=\mathbf{U}^{\top} \mathbf{F}\left(t, \mathbf{r}_{t}(\theta)\right)$,

keeping in mind $\mathbf{r}_{t}(\theta)=\mathbf{U} \mathbf{q}_{t}(\theta)$. In Eq. (20) $\xi_{l}$ and $\omega_{\mathrm{n}, l}$ are the damping ratio and the undamped natural frequency of the $l$ th mode. Equation (20) has a periodic stationary solution $\mathbf{q}_{\mathrm{p}}(t)=\mathbf{q}_{\mathrm{p}}(t+T)$ and it can be solved by boundary value problem. Considering the variation $\boldsymbol{\xi}(t)=\mathbf{U} \mathbf{u}(t)$ around the stationary solution

$\mathbf{q}(t)=\mathbf{q}_{\mathrm{p}}(t)+\mathbf{u}(t)$ and $\mathbf{r}(t)=\mathbf{r}_{\mathrm{p}}(t)+\boldsymbol{\xi}(t)$ 
the following linear so-called variational system can be formulated

$$
\begin{aligned}
& \ddot{\mathbf{u}}(t)+\left[2 \xi_{l} \omega_{\mathrm{n}, l}\right] \dot{\mathbf{u}}(t)+\left[\omega_{\mathrm{n}, l}^{2}\right] \mathbf{u}(t) \\
& =-\frac{1}{\cos \eta} \mathbf{U}^{\top} \sum_{i=1}^{Z} \int_{0}^{a_{\mathrm{p}}} g_{i}\left[\begin{array}{ccc}
\cos \varphi_{i} & \sin \varphi_{i} & 0 \\
-\sin \varphi_{i} & \cos \varphi_{i} & 0 \\
0 & 0 & 1
\end{array}\right] \mathbf{K}_{\mathrm{c}} \\
& \quad\left[\begin{array}{l}
\sin \varphi_{i} \cos \varphi_{i} \\
0]\left(\mathbf{U} \mathbf{u}(t)-\boldsymbol{\xi}\left(t-\tau_{i}\right)\right) \mathrm{d} z
\end{array}\right.
\end{aligned}
$$

After building a first order representation with using the definition $\mathbf{y}(t)=[\mathbf{u}(t) \dot{\mathbf{u}}(t)]^{\top}$ the system at (22) has the form

$\dot{\mathbf{y}}(t)=\mathbf{A y}(t)-\mathbf{B}(t) \mathbf{y}(t)+\sum_{i=1}^{Z} \mathbf{C}_{i}(t) \boldsymbol{\xi}\left(t-\tau_{i}\right)$,

where the matrices are

$$
\begin{aligned}
& \mathbf{A}=\left[\begin{array}{cc}
\mathbf{0} & \mathbf{I} \\
-\left[\omega_{\mathrm{n}, l}^{2}\right] & -\left[2 \xi_{l} \omega_{\mathrm{n}, l}\right]
\end{array}\right], \mathbf{B}(t)=\left[\begin{array}{cc}
\mathbf{0} & \mathbf{0} \\
\mathbf{c}(t) \mathbf{U} & \mathbf{0}
\end{array}\right], \\
& \mathbf{C}_{i}(t)=\left[\begin{array}{c}
\mathbf{0} \\
\mathbf{c}_{i}(t)
\end{array}\right], \mathbf{c}(t)=\sum_{i=1}^{Z} \mathbf{c}_{i}(t),
\end{aligned}
$$

using constant (zeroth order) values both to approximate the delayed terms and periodic coefficients as

$\mathbf{y}_{j+1}=\mathbf{A}_{\mathrm{e}} \mathbf{y}_{j}+\mathbf{D}_{j} \mathbf{y}_{j}-\sum_{i=1}^{Z} \mathbf{E}_{i, j} \boldsymbol{\xi}_{j-n_{i}}$

for the sake of simplicity with zeroth order approximation. In (27) $n_{i}=\left\lfloor\frac{\tau_{i}}{\Delta t}\right\rfloor$ and the matrices are

$\mathbf{D}_{j}=\left(\mathbf{I}-\mathbf{A}_{\mathrm{e}}\right) \mathbf{A}^{-1} \mathbf{B}_{j}$ and $\mathbf{E}_{i, j}=\left(\mathbf{I}-\mathbf{A}_{\mathrm{e}}\right) \mathbf{A}^{-1} \mathbf{C}_{i, j}$, where

$\mathbf{B}_{j}=\frac{1}{\Delta t} \int_{t_{j}}^{t_{j+1}} \mathbf{B}(t) \mathrm{d} t, \quad \mathbf{C}_{i, j}=\frac{1}{\Delta t} \int_{t_{j}}^{t_{j+1}} \mathbf{C}_{i}(t) \mathrm{d} t \quad$ and

$\mathbf{A}_{\mathrm{e}}=\mathrm{e}^{\mathbf{A} \Delta t}$ with $\quad \Delta t=t_{j+1}-t_{j}$.

The matrix exponential can be expressed analytically in the following form

$\mathbf{A}_{\mathrm{e}}=\left[\begin{array}{ll}\mathbf{A}_{\mathrm{e}, 11} & \mathbf{A}_{\mathrm{e}, 12} \\ \mathbf{A}_{\mathrm{e}, 21} & \mathbf{A}_{\mathrm{e}, 22}\end{array}\right], \quad$ where

$\mathbf{A}_{\mathrm{e}, 11}=\left[\begin{array}{ccc}\left(\cos \left(v_{1} \Delta t\right)-\frac{\alpha_{1}}{v_{1}} \sin \left(v_{1} \Delta t\right)\right) \mathrm{e}^{\alpha_{1} \Delta t} & \mathbf{0} \\ \mathbf{0} & \ddots & \\ & & \left(\cos \left(v_{m} \Delta t\right)-\frac{\alpha_{m}}{v_{m}} \sin \left(v_{m} \Delta t\right)\right) \mathrm{e}^{\alpha_{m} \Delta t}\end{array}\right]$,

$\mathbf{A}_{\mathrm{e}, 12}=\left[\begin{array}{ccc}\frac{1}{v_{1}} \sin \left(v_{1} \Delta t\right) \mathrm{e}^{\alpha_{1} \Delta t} & \mathbf{0} \\ & \ddots & \\ \mathbf{0} & & \frac{1}{v_{m}} \sin \left(v_{m} \Delta t\right) \mathrm{e}^{\alpha_{m} \Delta t}\end{array}\right]$,

$\mathbf{A}_{\mathrm{e}, 21}=\left[\begin{array}{ccc}-\frac{v_{1}^{2}+\alpha_{1}^{2}}{v_{1}} \sin \left(v_{1} \Delta t\right) \mathrm{e}^{\alpha_{1} \Delta t} & \mathbf{0} \\ \mathbf{0} & \ddots & -\frac{v_{m}^{2}+\alpha_{m}^{2}}{v_{m}} \sin \left(v_{m} \Delta t\right) \mathrm{e}^{\alpha_{m} \Delta t}\end{array}\right]$,

$\mathbf{A}_{\mathrm{e}, 22}=\left[\begin{array}{ccc}\left(\cos \left(v_{1} \Delta t\right)+\frac{\alpha_{1}}{v_{1}} \sin \left(v_{1} \Delta t\right)\right) \mathrm{e}^{\alpha_{1} \Delta t} & \mathbf{0} \\ & \ddots & \\ 0 & & \left(\cos \left(v_{m} \Delta t\right)+\frac{\alpha_{m}}{v_{m}} \sin \left(v_{m} \Delta t\right)\right) \mathrm{e}^{\alpha_{m} \Delta t}\end{array}\right]$.

$\mathbf{c}_{i}(t)=\frac{1}{\cos \eta} \mathbf{U}^{\top} \int_{0}^{a_{\mathrm{p}}} g_{i}\left[\begin{array}{ccc}\cos \varphi_{i} & \sin \varphi_{i} & 0 \\ -\sin \varphi_{i} & \cos \varphi_{i} & 0 \\ 0 & 0 & 1\end{array}\right]$

$\mathbf{K}_{\mathrm{c}}\left[\sin \varphi_{i} \cos \varphi_{i} 0\right] \mathrm{d} z$.

Note that matrices $\mathbf{B}(t)=\mathbf{B}(t+T)$ and $\mathbf{C}_{i}(t)=\mathbf{C}_{i}(t+$ $T$ ) are time periodic with the possible smallest period $T$ which (is in fact) can be the integer quotient of the theoretical tooth passing period $T_{Z}=\frac{2 \pi}{Z \Omega}$ depending on the geometrical allocation of the pitch angles $\varphi_{\mathrm{p}, i}$. Also the number of delays at (23) depends on the distribution of pitch angles, but it kept to be the maximum amount $Z$ in order to ease the notation.

With the formula presented in (23) we can calculate the new state at different time steps by semi-discretization [28] where $v_{i}$ is the damped natural frequency of the $i$ th natural mode, with $\alpha_{i}$ its damping exponent, so that

$\alpha_{l}=-\xi_{l} \omega_{\mathrm{n}, l}, \quad \nu_{l}^{2}=\omega_{\mathrm{n}, l}^{2}-\alpha_{l}^{2}$

The matrix $\mathbf{A}_{\mathrm{e}}$ is constant for all the process if $t_{j}(j=$ $1, \ldots, r)$ time mesh is equidistant, while the matrices $\mathbf{D}_{j}$ and $\mathbf{E}_{i, j}$ vary. For each time step it is necessary to calculate and store the matrices $\mathbf{A}_{\mathbf{e}}+\mathbf{D}_{j}$ and $\mathbf{E}_{i, j}$. This set of matrices represents the step matrices for the time step.

When the tool has helix angle, an integration of the matrices along the helix has to be done, which can be done numerically by dividing the depth of cut into sufficiently small parts replacing integral presented in (18) with summation. Also, in the same case of the helical flutes, and when there are more 


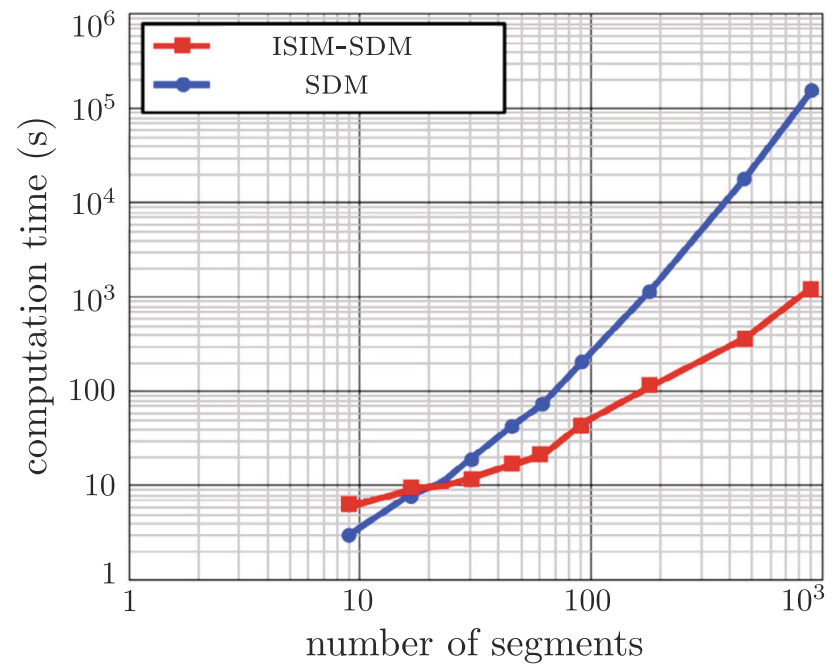

Fig. 2 Computation time by optimised SDM and by the (ISIM)

than one tooth cutting simultaneously, when calculating the matrices for some angular positions there will be some teeth, or parts of a tooth, which are cutting at only one portion of the segment.

With the semi-discretization method presented at (27) the implicit subspace iteration (ISIM-SDM) new state for Krylov subspace or for power iteration can be determined without determining the transition matrix $\mathbf{Z}$ performing the following iteration $r=T / \Delta t$ times knowing that in any other iterations the matrices at (28), (29), (30), (31) are available.

\subsection{Calculation time comparison}

Figure 2 shows a comparison of the time required for calculation of the stability lobes in similar conditions by the optimized SDM and by the subspace iteration, depending on the number of segments in which the cutting arc is divided. It becomes clear that for a high number of segments the subspace iteration is much more favorable. Therefore, this method permits the evaluation for uneven pitch tools, requiring $Z$ times more segments, $Z$ being the number of teeth of the tool.

\subsection{Calculation of the step matrices for uneven pitch tools}

For the general case of uneven pitch tools the period to consider is no more one tooth pass, but the integer multiple of tooth pass or in general maximum the complete rotation of the tool. Therefore, the number of segments to consider is larger than for regular pitch tools.

This is almost the only change to consider if the resolution is done by the subspace iteration method. A larger number of step matrices have to be considered and stored, and for each vector iteration a higher number of operations with those step matrices have to be performed. The size of the time step does not change, as it depends on the highest natural frequency considered and on the rotation speed; therefore, the ratio of the time of calculation for uneven pitch with respect to the corresponding regular pitch case should be linearly proportional to the number of teeth. It is interesting to remind that for a conventional SDM resolution, the time would vary roughly with the exponent 3 of the number of teeth, similarly presented at [29].

\section{Comparison with already reported cases: Sellmeier and Denkena}

The validation of the methodology presented in this paper to obtain the stability diagrams will be validated by comparison to previous works based on other methods. The paper by Sellmeier and Denkena [25] presented the methodology to obtain stability diagrams with uneven pitch mills by the SDM, including some experimental verifications. One of the processes proposed in that paper was used contrasting the results of the method presented here. The parameters are summarized in Table 1.

There is very good agreement of the results obtained with the method of subspace iteration and the results obtained by the paper mentioned for the 4 pitch angle variations proposed. The panels in Fig. 3 show the results obtained for each of the tools proposed.

Apart from the stable islands, it is interesting to see that the stability of the system is not improved by any of the pitch variations proposed in the paper by Sellmeier and Denkena [25] at the minimum of the e.g. second lobe. An alternative pitch angle combinations following a linear evolution proposed by Budak [1] were tested for the rotation speed 2,000 $\mathrm{rev} / \mathrm{min}$, at the minimum of the second lobe. As seen in Fig. 4, the stable depth of cut is increased in $41 \%$ at that speed. But that tool produces less stable cut for all the velocities except exactly for the velocities in the vicinity of the one selected for the optimization.
Table 1 Milling process parameters in the example chosen from [25]

\begin{tabular}{ll}
\hline System & $m=5.1 \mathrm{~kg}, c=468.8 \frac{\mathrm{Ns}}{\mathrm{m}}, k=10.4 \frac{\mathrm{N}}{\mu \mathrm{m}}, \xi=3.3 \%, \omega_{\mathrm{n}}=227.7 \mathrm{~Hz}$, direction: $x$, \\
Tool & Conventional, cylindrical, $D=20 \mathrm{~mm}, Z=4, \eta=30^{\circ}$, \\
Material & Aluminum, $K_{\mathrm{c}, t}=793.99 \frac{\mathrm{N}}{\mathrm{mm}^{2}}, K_{\mathrm{c}, r}=109.41 \frac{\mathrm{N}}{\mathrm{mm}^{2}}$, \\
Process & Full immersion, theoretical feed per tooth $f_{Z}=0.12 \mathrm{~mm}$, feed direction: $x$ \\
\hline
\end{tabular}


Fig. 3 Results of the example by [25] for pitch angles $\mathbf{a}$ $\varphi_{\mathrm{p}, i}=80^{\circ}, 100^{\circ}, 80^{\circ}, 100^{\circ}$, b $\varphi_{\mathrm{p}, i}=70^{\circ}, 110^{\circ}, 70^{\circ}, 110^{\circ}$, c $\varphi_{\mathrm{p}, i}=75^{\circ}, 105^{\circ}, 75^{\circ}, 105^{\circ}$ and $\mathbf{d} \varphi_{\mathrm{p}, i}=85^{\circ}, 95^{\circ}, 85^{\circ}$, $95^{\circ}$. Dashed lines denotes uniform tool with $\varphi_{\mathrm{p}, i}=90^{\circ}$ pitches
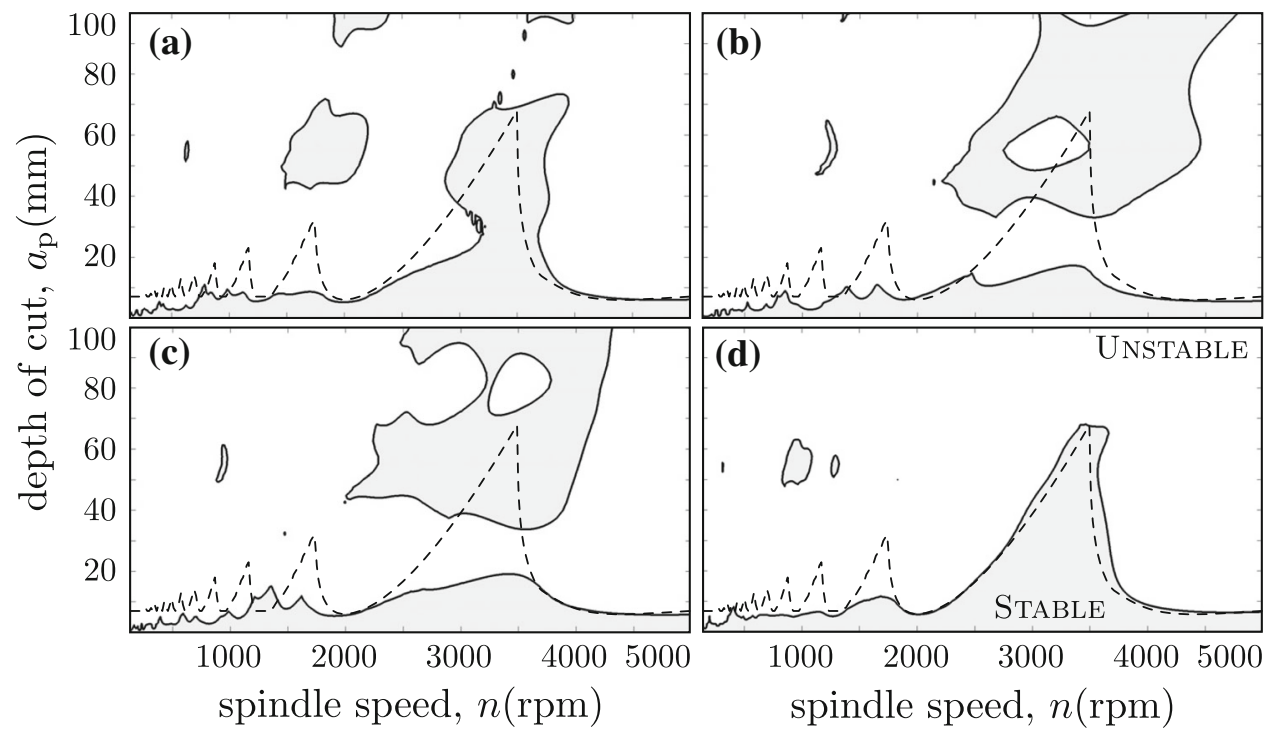

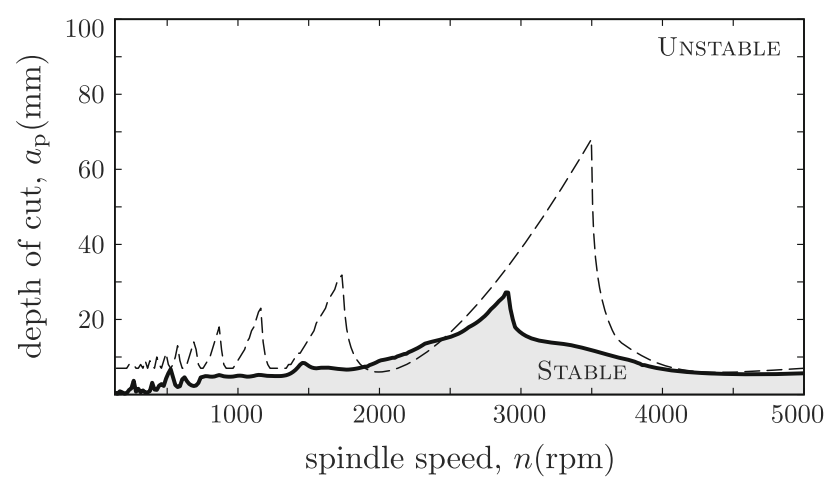

Fig. 4 Results of the example by Sellmeier and Denkena [25] (dashed) for pitch angles after Budaks [1] method results $\varphi_{\mathrm{p}, i}=$ $70.23^{\circ}, 83.4^{\circ}, 96.59^{\circ}, 109.77^{\circ}$ (continuous). Dashed lines denotes uniform tool with $\varphi_{\mathrm{p}, i}=90^{\circ}$ pitches

It might be interesting to mention that in the low order lobes region it should be difficult to produce stabilization by irregular pitch due to the large angle variation required. It seems that most of the times the selection of a different rotation velocity at the sweet zone would be preferable.

\section{Assessment of the pitch variation optimization by Budak}

Budak [1,2] presented the first formulation for selection of the pitch angles of uneven pitch mills to avoid chatter at determined rotation speeds. One of the possible formulations shown consists in selecting linearly varying pitch angles, with the variation calculated after the ratio between the dominant natural frequency and the rotation frequency. The development is based in the frequency domain, and the harmonics of the dominant chatter vibration frequency are not included in the formulation. Therefore, it can be interesting to see if the

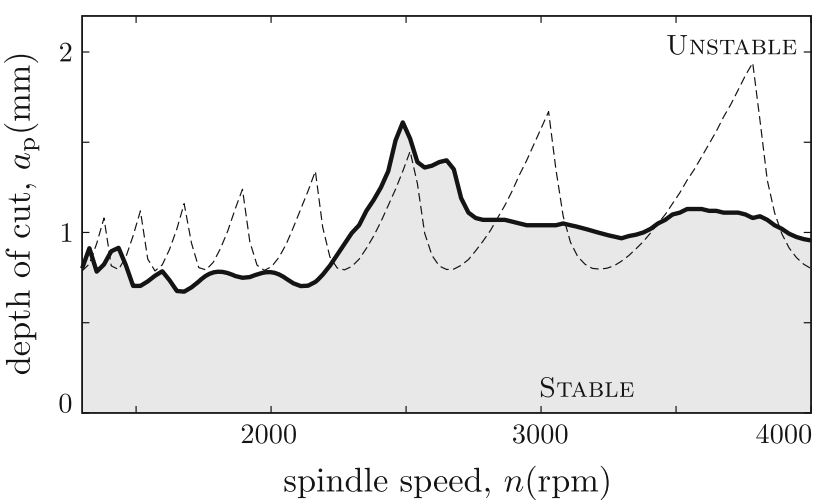

Fig. 5 Results of the example by Budak [2] for pitch angles $\varphi_{\mathrm{p}, i}=$ $78^{\circ}, 86^{\circ}, 94^{\circ}, 102^{\circ}$ (continuous) and its conventional counterpart (dashed)

simplifications assumed for the development affect the result in the foreseen stability increase.

One of the examples shown in [2] consists in a process of milling magnesium. The dominant mode is due to the tool assembly. That mode has components both in $x$ and $y$ directions, with slightly varying natural frequency for each direction, or it might also be that there are two modes with different natural frequencies. No values for the cutting force coefficients were given in the paper, as the goal was to increase the stable cutting depth in a practical case.

The important fact in that example was that an increase of stability was obtained by using a determined non constant pitch tool, with pitch angles $\varphi_{\mathrm{p}, i}=78^{\circ}, 86^{\circ}, 94^{\circ}, 102^{\circ}$. These angles were calculated after the design process shown in [1] for the natural angular frequency $6000 \mathrm{rad} / \mathrm{s}$ and at the rotation velocity $2,500 \mathrm{rev} / \mathrm{min}$.

From Fig. 5, it follows that the pitch angle combination proposed by Budak beautifully finds a sweet area at the 
Fig. 6 Stability diagrams at high order lobes for tools optimised at 600, 400 and 800 rpm. (blue $\varphi_{\mathrm{p}, i}=90^{\circ}$, red $\varphi_{\mathrm{p}, i}=88.586^{\circ}, 89.529^{\circ}$, $90.471^{\circ}, 91.414^{\circ}$, green $\varphi_{\mathrm{p}, i}=$ $89.058^{\circ}, 89.686^{\circ}, 90.314^{\circ}$, $90.942^{\circ}$, purple $\varphi_{\mathrm{p}, i}=$ $88.115^{\circ}, 89.372^{\circ}, 90.629^{\circ}$, $\left.91.886^{\circ}\right)$. (Color figure online)

Fig. 7 Stability diagrams at high order lobes for tools optimized at 2,500 rpm inducing $\varphi_{\mathrm{p}, i}=78^{\circ}, 86^{\circ}, 94^{\circ}, 102^{\circ}$ (continuous)
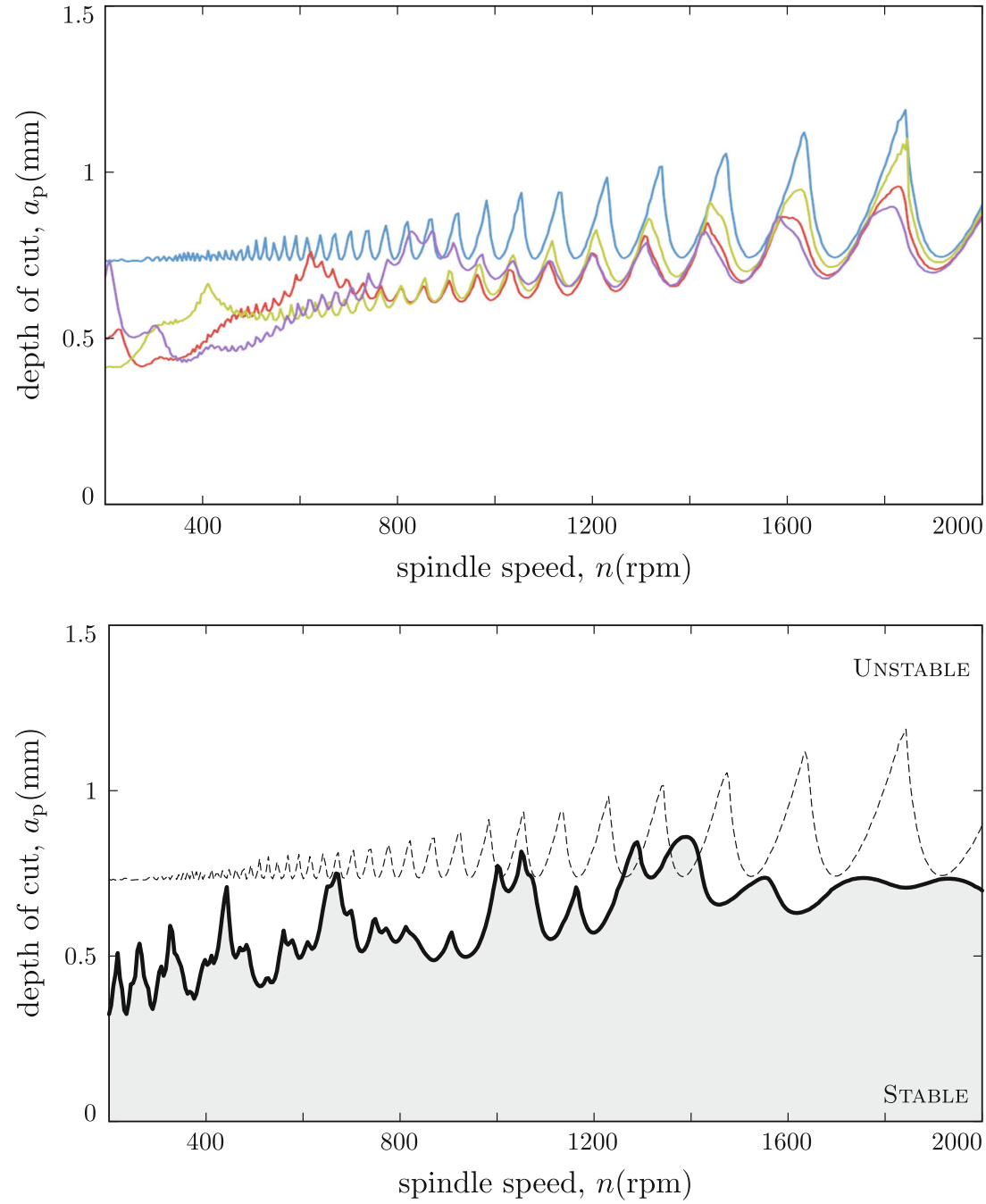

selected velocity region. In this case, the lobe order at which the sweet zone is found is 6 . The rotation frequency is $42 \mathrm{~Hz}$, and the bandwidth at the mode considered is roughly $36 \mathrm{~Hz}$ (=damping ratio times natural frequency). That means that in this velocity region, the harmonics of the main vibration frequency does not produce very large dynamic response at the system. Therefore, it would be interesting to analyze the behavior at lower rotation speeds, where that speed produces harmonics inside the bandwidth of the dominant mode.

For that analysis, a lower velocity region was selected. The analysis was performed in the region from 200 to 2,000 rpm, which already represents high order lobes and the practical impossibility to calculate the stability diagram by time domain methods. The diagrams were calculated by the ISIM-SDM, and the tools were designed after Budak [2] to give optimum stability at the rotation velocities 600 $\operatorname{rpm}\left(\varphi_{\mathrm{p}, i}=88.586^{\circ}, 89.529^{\circ}, 90.471^{\circ}, 91.414^{\circ}\right), 400 \mathrm{rpm}$ $\left(\varphi_{\mathrm{p}, i}=89.058^{\circ}, 89.686^{\circ}, 90.314^{\circ}, 90.942^{\circ}\right)$, and $800 \mathrm{rpm}$ $\left(\varphi_{\mathrm{p}, i}=88.115^{\circ}, 89.372^{\circ}, 90.629^{\circ}, 91.886^{\circ}\right)$. The results are shown in Fig. 6.
From this figure it follows that each irregular pitch tool has local optimum behaviour at the speed region for which it was designed, following Budaks methodology [2], but the stability tends to be lower than when using the regular pitch mill. As a comparison, the stability diagram produced for the tool optimized for $2,500 \mathrm{rpm}$, that is, a tool with much larger angle variation, is shown in Fig. 7. It follows that this tool does not produce stability benefits in the high order lobe region.

\section{Conclusions}

As a general conclusion, the ISIM enables the accurate calculation of stability diagrams at high order lobes when using irregular pitch mills. The method combined by semidiscretization (ISIM-SDM) was validated by comparison with results previously published at low order lobe region. The pitch angle selection proposed by Budak [1,2] finds sweet regions at the desired velocities, although at high order 
lobes regions the stability seems to deteriorate when compared to that of the regular pitch mill. As a research to be developed, the possibility of increasing the stability by the use of irregular pitch mills in the high order lobe region has to be analyzed. Another field of development would be the analysis of the design method proposed by Suzuki et al. [3] for the optimization of tools when there are two modes involved in the vibration.

Acknowledgments This research was partially supported by the Hungarian Scientific Research Foundation OTKA Grant No. K108779 and IK4-Ideko Research Alliance.

\section{References}

1. Budak E (2003) An analytical design method for milling cutters with nonconstant pitch to increase stability, part I: theory. J Manuf Sci Eng 125(1):29-35

2. Budak E (2003) An analytical design method for milling cutters with nonconstant pitch to increase stability, part II: theory. J Manuf Sci Eng 125(1):35-38

3. Suzuki N, Kojima T, Hino R, Shamoto E (2012) A novel design method of irregular pitch cutters to attain simultaneous suppression of multi-mode regenerations. Procedia CIRP 4:98-102

4. Tobias S, Fishwick W (1958) Theory of regenerative machine tool chatter. Engineer 205:199-203

5. Tlusty J, Polacek M (1957) Beispiele der Behandlung der selbsterregten Schwingung der Werkzeugmaschinen. FoKoMa, Hanser Verlag, Munich

6. Merritt H (1965) Theory of self-excited machine tool chatter. Trans ASME J Eng Ind 87:447-454

7. Opitz H, Bernardi F (1970) Investigation and calculation of chatter behaviour of lathes and milling machines. CIRP Ann 18:335-342

8. Minis I, Yanushevsky T, Tembo R, Hocken R (1990) Analysis of linear and nonlinear chatter in milling. Ann CIRP 39:459-462

9. Floquet MG (1883) Équations différentielles linéaires, a coefficients périodiques. Journal de Crelle 12:47-88

10. Budak E, Altintas Y (1998) Analytical prediction of chatter stability conditions for multidegree of freedom systems in milling part I: general formulation, part II: application of the general formulation to common milling systems. Trans ASME J Eng Ind 120:22-36

11. Altintas Y, Budak E (1995) Analytical prediction of stability lobes in milling. CIRP Ann Manuf Technol 44:357-362

12. Davies M, Pratt J, Dutterer B, Burns T (2000) The stability of low radial immersion milling. CIRP Ann Manuf Technol 49(1):37-40
13. Insperger T, Stepan G (2002) Semi-discretization method for delayed systems. Int J Numer Methods Eng 55:503-518

14. Insperger T, Mann BP, Stepan G, Bayly PV (2003) Stability of upmilling and down-milling, part 1: alternative analytical methods. Int J Mach Tools Manuf 43(1):25-34

15. Bayly P, Halley J, Mann B, Davies M (2003) Stability of interrupted cutting by temporal finite element analysis. J Manuf Sci Eng 125(2):220-225

16. Zatarain M, Bediaga I, Munoa J, Insperger T (2010) Analysis of directional factors in milling: importance of multi-frequency calculation and of the inclusion of the effect of the helix angle. Int $\mathbf{J}$ Adv Manuf Technol 47(5):535-542

17. Salje E, Gerloff H, Meyer J (1988) Comparison of machine tool elements made of polymer concrete and cast iron. CIRP Ann Manuf Technol 37(1):381-384

18. Den Hartog J (1934) Mechanical vibrations. McGraw-Hill Book Company, New York

19. Ema S, Marui E (2000) Suppression of chatter vibration of boring tools using impact dampers. Int J Mach Tools Manuf 40(8):11411156

20. Jemielniak K, Widota A (1984) Suppression of self-excited vibration by the spindle speed variation method. Int J Mach Tool Des Res 24(3):207-214

21. Slavicek J (1965) The effect of irregular tooth pitch on stability of milling. In: Proceedings of the 6th MTDR Conference, Pergamon Press, London, pp 15-22

22. Opitz H, Dregger EU, Roese H (1966) Improvement of the dynamic stability of the milling process by irregular tooth pitch. Proc Adv MTDR Conf 7:213-227

23. Vanherck $P$ (1967) Increasing milling machine productivity by use of cutters with non-constant cutting edge pitch. 8th MTDR Conference, Manchester, pp 947-960

24. Tlusty J, Ismail F, Zaton W(1983) Use of special milling cutters against chatter. NAMRC 11, University of Wisconsin, SME, pp 408-415

25. Sellmeier V, Denkena B (2011) Stable islands in the stability chart of milling processes due to unequal tooth pitch. Int $\mathrm{J}$ Mach Tools Manuf 51(2):152-164. doi:10.1016/j.ijmachtools.2010.09.007

26. Otto A, Radons G (2013) Application of spindle speed variation for chatter suppression in turning. CIRP J Manuf Sci Technol 6(2):102-109

27. Shampine LF, Thompson S (2000) Solving delay differential equations with dde23. Appl Numer Math 37(4):441-458

28. Insperger T, Stepan G (2011) Semi-discretization for time-delay systems: stability and engineering applications. Springer, New York

29. Henninger C, Eberhard P (2008) Improving the computational efficiency and accuracy of the semi-discretization method for periodic delay-differential equations. Eur J Mech A 27(6):975-985 